\title{
Floppy Modes and Nonaffine Deformations in Random Fiber Networks
}

\author{
Claus Heussinger and Erwin Frey \\ Arnold Sommerfeld Center for Theoretical Physics and Center for NanoScience, Department of Physics, \\ Ludwig-Maximilians-Universität München, Theresienstrasse 37, D-80333 München, Germany \\ (Received 26 March 2006; published 8 September 2006)
}

\begin{abstract}
We study the elasticity of random fiber networks. Starting from a microscopic picture of the nonaffine deformation fields, we calculate the macroscopic elastic moduli both in a scaling theory and a selfconsistent effective medium theory. By relating nonaffinity to the low-energy excitations of the network ("floppy modes"), we achieve a detailed characterization of the nonaffine deformations present in fibrous networks.
\end{abstract}

DOI: 10.1103/PhysRevLett.97.105501

PACS numbers: $62.25 .+\mathrm{g}, 81.05 . \mathrm{Lg}, 87.16 . \mathrm{Ka}$

Materials as different as granular matter, colloidal suspensions, or lithospheric block systems share the common property that they may exist in a highly fragile state $[1,2]$. While, in principle, able to withstand static shear stresses, small changes in the loading conditions may lead to large scale structural rearrangements or even to the complete fluidization of the material [2-4]. To understand the extraordinary mechanical properties of these systems, new concepts have to be developed that go beyond the application of classical elasticity theory and that sufficiently reflect the presence of the microstructure [5]. One example is the "stress-only" approach to the elasticity of granular materials [6], where the elimination of the kinematic degrees of freedom accounts for the infinite stiffness of the grains. This seems to capture the inhomogeneous distribution of stresses in the sample and their concentration along the so-called force chains [7]. In jammed systems of soft spheres, on the other hand, fragility has recently been shown to directly affect the deformation response of the system. While it may induce anomalous deformation fields that strongly deviate from the expectations of homogeneous elasticity ("nonaffine" deformations) [8], it may also lead to a proliferation of low-frequency vibrational states far beyond the usual Debye behavior of ordinary solids [9]. It has been argued that these low-energy vibrations derive from a set of zero-frequency modes (floppy modes) that are present just below the jamming threshold [10] and relate to the ability of the structure to internally rearrange without any change in its potential energy. This concept of floppy modes has also been used in connection with elastic percolation networks where the fragile state is reached by diluting a certain fraction of nearest-neighbor contacts. In these systems, constraint-counting arguments may be used to determine the percolation transition at which the system loses its rigidity [11].

Here our focus is on a particular class of heterogeneous networks composed of cross-linked fibers. These systems have recently been suggested as model systems for studying the mechanical properties of paper sheets [12] or biological networks of semiflexible polymers $[13,14]$.
While these networks are known to have a rigidity percolation transition at low densities $[15,16]$, we show here that even networks in the high-density regime in many ways resemble the behavior of fragile matter, despite the fact that they are far away from the percolation threshold. We identify the relevant floppy modes and highlight their importance for understanding the macroscopic elasticity of the network. In particular, we will be able to explain the occurrence of an anomalous intermediate scaling regime observed in recent simulations $[15,17,18]$. In this regime, the shear modulus was found to depend on density (measured relative to the percolation threshold) as $G \sim \delta \rho^{\mu}$ with a fractional exponent as large as $\mu \approx 6.67$ [15]. Also, highly nonaffine deformations $[17,19]$ as well as inhomogeneous distribution of stresses in the network have been found. Heuristic nonaffinity measures have been devised $[17,19]$; however, little is known about the actual nature of the deformations present. While the expression "nonaffine" is exclusively used to signal the absence of conventional homogeneous elasticity, scarce positive characterization of nonaffine deformations has been achieved up to now [20]. This Letter tries to fill this gap by characterizing in detail the nonaffine deformation field present in fibrous networks. By relating nonaffinity to the floppy modes of the structure, we can, starting from a microscopic picture, calculate the macroscopic elastic moduli in both a scaling theory and a self-consistent effective medium theory. In analogy with the affine theory of rubber elasticity for flexible polymer gels, our approach might very well serve as a second paradigm to understand the elasticity of microstructured materials. Because of the proximity to the fragile state, it might also be of relevance to force transmission in granular media and to the phenomenon of jamming.

The two-dimensional fiber network we consider is defined by randomly placing $N$ elastic fibers of length $l_{f}$ on a plane of area $A=L^{2}$ such that both the position and orientation are uniformly distributed. We consider the fiber-fiber intersections to be perfectly rigid but freely rotatable cross-links that do not allow for relative sliding 
of the filaments. The randomness entails a distribution of angles $\theta \epsilon[0, \pi]$ between two intersecting filaments

$$
P(\theta)=\frac{\sin (\theta)}{2},
$$

while distances between neighboring intersections, the segment lengths $l_{s}$, follow an exponential distribution [21]

$$
P\left(l_{s}\right)=\left\langle l_{s}\right\rangle^{-1} e^{-l_{s} /\left\langle l_{s}\right\rangle} .
$$

The mean segment length $\left\langle l_{s}\right\rangle$ is inversely related to the line density $\rho=N l_{f} / A$ as $\left\langle l_{s}\right\rangle=\pi / 2 \rho$. The segments are modeled as classical beams with cross-section radius $r$ and bending rigidity $\kappa$. Loaded along their axis ("stretching"), such slender rods have a rather high stiffness $k_{\|}\left(l_{s}\right)=4 \kappa / l_{s} r^{2}$, while they are much softer with respect to transverse deformations $k_{\perp}\left(l_{s}\right)=3 \kappa / l_{s}^{3}$ ("bending"). Numerical simulations for the effective shear modulus $G$ of this network have identified a crossover scaling scenario characterized by a length scale $\xi=l_{f}\left(\delta \rho l_{f}\right)^{-\nu}$ and $\nu \approx$ 2.84 [15] that mediates the transition between two drastically different elastic regimes. For fiber radius $r \gg \xi$, the system is in an affine regime where the elastic response is dominated mainly by stretching deformations homogeneously distributed throughout the sample. The modulus in this regime is simply proportional to the typical stretching stiffness $G_{\text {aff }} \propto k_{\|}\left(\left\langle l_{s}\right\rangle\right)$ and independent of the fiber length $l_{f}$. This is in marked contrast to the second regime at $r \ll$ $\xi$. There, only nonaffine bending deformations are excited, and the modulus shows a strong dependence on fiber length $G_{\text {na }} \propto k_{\perp}\left(\left\langle l_{s}\right\rangle\right)\left(l_{f} /\left\langle l_{s}\right\rangle\right)^{\mu-3}$. Using renormalization-group language, the parameters $r$ and $l_{f}$ may be viewed as scaling fields (measured in units of the "lattice constant" $\left.\left\langle l_{s}\right\rangle\right)$. The stretching dominated regime may then be characterized by an (affine) fixed point at $l_{f} \rightarrow \infty$ and finite radius $r \neq 0$. On the other hand, the (nonaffine) fixed point of the bending dominated regime is obtained by first letting $r \rightarrow 0$ and then performing $l_{f} \rightarrow \infty$. This suggests that the elastic properties in the latter regime may be analyzed at vanishing radius $r=0$, that is, by putting the system on the stable manifold of the fixed point.

In the following, we will exploit this limit to calculate the modulus $G_{\text {na }}$ in the nonaffine regime. Central to the analysis is the recognition that in this limit the ratio of bending to stretching stiffness $k_{\perp} / k_{\|} \propto r^{2}$ tends to zero and bending deformations become increasingly soft. We thus obtain the much simpler problem of a central-force network. However, as only two fibers may intersect at a cross-link, the coordination is $z<4$ [22] and rigid regions may not percolate through the system $[23,24]$. This implies that, on a macroscopic level, the elastic moduli will be zero, while microscopically displacements can be chosen such that segment lengths need not be changed. These are the floppy modes of the structure that entail the fragility of the network in the bending dominated regime. It has been argued that a critical coordination of $z_{c}=4$ is necessary to give the network rigidity [24]. This value defines the "isostatic" point, which in our network corresponds to taking the limit $l_{f} \rightarrow \infty$. Thus, we arrive at the conclusion that isostaticity and the onset of rigidity seem to be intimately connected to the fixed point governing the nonaffine regime. While it is usually not possible to deduce the specific form of the floppy modes, the fibrous architecture allows for their straightforward construction (see Fig. 1). In a first step, we perform an arbitrary axial displacement $\delta z$ of a given (primary) fiber as a whole. This, of course, will also affect the crossing (secondary) fibers such that the lengths of interconnecting segments change. In a second step, therefore, one has to account for the length constraints on these segments by introducing cross-link deflections $\bar{y}_{i}=$ $-\delta z \cot \theta_{i}$ transverse to the contour of the primary fiber. As a result, all segment lengths remain unchanged to first order in $\delta z$ [25]. The construction is, therefore, suitable to describe the linear response properties of the network, while at the same time it offers an explanation for the stiffening behavior found in fully nonlinear simulations $[19,26]$. Any finite strain necessarily leads to the energetically more expensive stretching of bonds and, therefore, to an increase of the modulus.

The identified modes take the form of localized excitations that affect only single filaments and their immediate surroundings. By superposition, we may therefore construct a displacement field that allows the calculation of macroscopic quantities such as the elastic moduli. To achieve this, we need to know the typical magnitude of displacements $\delta z$ of a given fiber relative to its surroundings, the crossing secondary fibers. Since $\delta z$ is defined on the scale of the complete fiber, we do not expect any dependence on average segment length $\left\langle l_{s}\right\rangle$, such that $\delta z \propto$ $l_{f}$ remains as the only conceivable possibility. Alternatively, one may obtain the same result by assuming that the individual fiber centers follow the macroscopic strain

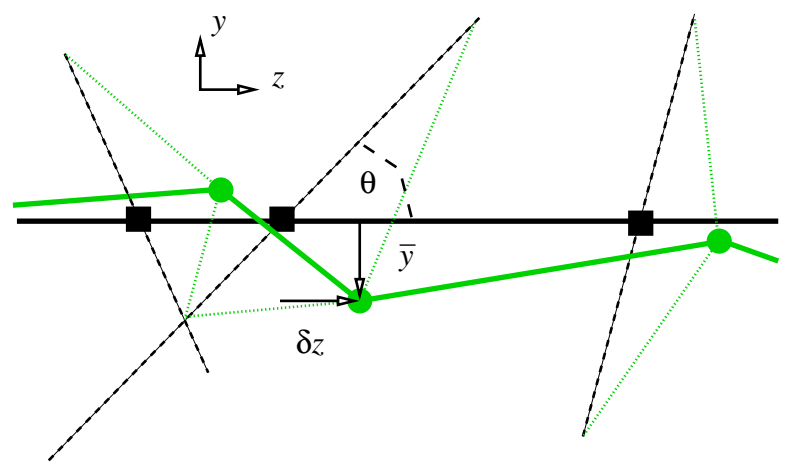

FIG. 1 (color online). Construction of a floppy mode by axial displacement $\delta z$ of the primary fiber (drawn horizontally) and subsequent transverse deflection $\bar{y}=-\delta z \cot \theta$ of the cross-links to restore the segment lengths on the secondary fibers (dashed lines, possible to first order in $\delta z$ ). Initial cross-link positions are marked as black squares, final configurations as green circles. 
field in an affine way. Then relative displacements of centers of neighboring fibers would be proportional to their typical distance. This is of the order filament length $l_{f}$ and again $\delta z \propto l_{f}$. Note, however, that the assumption of affine displacement of the fiber centers cannot be literally true for fibers intersecting at very small angles $\theta \rightarrow 0$. To avoid a diverging transverse deflection $\bar{y}_{i}=-\delta z \cot \theta \rightarrow \infty$, the two fibers will most likely not experience any relative motion at all and $\delta z \rightarrow 0$. Truly affine displacements can, therefore, be established only on scales larger than the filament length. It should also be clear that the assumption of affine displacements of the fiber centers is different from the usual approach of assigning affine deformations on the scale of the single segment. The latter would lead to deformations $\delta_{\text {aff }} \propto l_{s}$, proportional to the length $l_{s}$ of the segment. Instead, axial displacements of the fiber as a whole are, by construction of the floppy mode, directly translated into nonaffine deformations $\delta_{\text {na }} \propto l_{f}$, which do not depend on the length of the segment.

Restoring the radius $r$ to its finite value, the floppy modes acquire energy and lead to bending of the fibers. A segment of length $l_{s}$ will then typically store the energy $w_{b}\left(l_{s}\right) \simeq \kappa \delta_{\mathrm{na}}^{2} / l_{s}^{3} \simeq \kappa l_{f}^{2} / l_{s}^{3}$. By averaging over the segment length distribution Eq. (2), one may calculate the average bending energy $\left\langle W_{b}\right\rangle$, stored in a fiber consisting of $n \simeq \rho l_{f}$ segments,

$$
\left\langle W_{b}\right\rangle \simeq \rho l_{f} \int_{l_{\min }}^{\infty} d l_{s} P\left(l_{s}\right) \frac{\kappa \delta_{\mathrm{na}}^{2}}{l_{s}^{3}}
$$

We assume the integral to be regularized by a lower cutoff length $l_{\min }$ that we now determine in a self-consistent manner. Physically, $l_{\min }$ corresponds to the shortest segments along the fiber that contribute to the elastic energy. Even though we know [see Eq. (2)] that arbitrarily short segments do exist, their high bending stiffness $k_{\perp}\left(l_{s}\right) \propto l_{s}^{-3}$ makes their deformation increasingly expensive. Segments with length $l_{s}<l_{\min }$ will, therefore, be able to relax from their floppy mode deformation $\delta_{\text {na }}$, thereby reducing their bending energy from $w_{b}\left(l_{\text {min }}\right)$ to nearly zero. However, due to the length constraints, this relaxation necessarily leads to the movement of an entire secondary fiber and to the excitation of a floppy mode there. By balancing $w_{b}\left(l_{\min }\right)=$ $\left\langle W_{b}\right\rangle$, this gives $l_{\min } \simeq 1 / \rho^{2} l_{f}$ and for the average bending energy of a single fiber $\left\langle W_{b}\right\rangle \simeq \kappa / l_{f}\left(\rho l_{f}\right)^{6}$. This implies for the modulus $G_{\mathrm{na}} \simeq \rho / l_{f}\left\langle W_{b}\right\rangle \propto \rho^{7}$, which compares well with the simulation result of $\mu=6.67$. What is more, by equating the energy $\left\langle W_{b}\right\rangle$ with $\left\langle W_{s}\right\rangle \simeq \kappa l_{f} r^{-2}$ valid in the affine stretching regime, one can also infer the crossover exponent $\nu=3$.

In summary, we have succeeded in explaining the elasticity of the bending dominated regime starting from the microscopic picture of the floppy modes that characterize directly the deformation field deep inside the nonaffine regime. Alternatively, one might try to understand the emergent nonaffinity in a perturbative approach that considers deviations from an affine reference state. Such a line of reasoning has recently been suggested in Ref. [18], where nonaffine boundary layers, growing from the filament ends, are assumed to perturb the perfect affine order. However, comparing with their simulation data, the authors could not confirm the scaling picture unambiguously and acknowledged the need for further numerical as well as improved theoretical work [18]. Thus, nonaffine elasticity in fibrous networks appears to be intrinsically a nonperturbative strong-coupling phenomenon for which the floppy mode picture provides the correct low-energy excitations. As we will explicitly show next, one particular strength of our approach is that the scaling picture can readily be extended to a full theory that self-consistently calculates the modulus in a nonaffine effective medium theory.

To set up the theory, we consider a single filament together with its cross-links that provide the coupling to the medium. The energy of this assembly consists of two parts. First, the bending energy of the primary fiber

$$
W_{b}[y(z)]=\frac{\kappa}{2} \int\left(\frac{\partial^{2} y}{\partial z^{2}}\right)^{2} d z
$$

due to a transverse deflection $y(z)$. A second stretching energy contribution arises whenever a cross-link deflection $y_{i}=y\left(z_{i}\right)$ differs from its prescribed value $\bar{y}_{i}=-\delta z \cot \theta_{i}$ and may be written in the form of an harmonic confining potential $W_{s}\left(y_{i}\right)=\frac{1}{2} k_{i}\left(y_{i}-\bar{y}_{i}\right)^{2}$ that acts individually on each of the $n \simeq \rho l_{f}$ cross-links. It allows the filament to reduce its own energy at the cost of deforming the elastic matrix into which it is imbedded. Performing a configura-

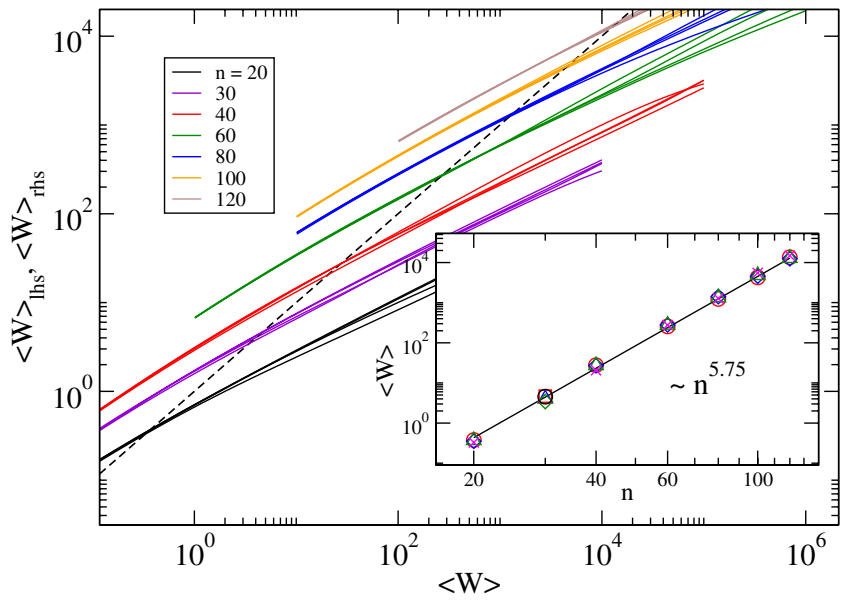

FIG. 2 (color online). Graphical solution of Eqs. (5) and (6) for various numbers $n$ of cross-links obtained by calculating the intersection between the left side of the equation $\langle W\rangle_{\mathrm{lhs}}$ (bisecting line, dashed curve) with the right side $\langle W\rangle_{\text {rhs }}$ (solid curves). The different curves for a given $n$ correspond to ensembles of varying size. They seem to diverge in the limit $\langle W\rangle_{\mathrm{rhs}} \gg\langle W\rangle_{\mathrm{lhs}}$. In fact, there (and only there) the averaging procedure is illdefined [26]. Inset: Resulting dependence of $\langle W\rangle$ on $n$. 
tional average $\langle$.$\rangle over cross-link positions z_{i}$ and orientations $\theta_{i}$, we obtain the average elastic energy stored in a single fiber as

$$
\langle W\rangle=\left\langle\min _{y(z)}\left(W_{b}[y(z)]+\sum_{i=1}^{n} \frac{k_{i}}{2}\left(y_{i}-\bar{y}_{i}\right)^{2}\right)\right\rangle .
$$

To solve the model, we further need to specify the stiffness $k_{i}=k\left(\theta_{i}\right)$ of the medium that relates to the relaxation mode of a cross-link on the primary filament from its floppy mode deflection. As we have argued above, any relaxation of this kind must act as axial displacement on a secondary fiber, thus exciting a new floppy mode there. The energy scale associated with this is $\langle W\rangle$ such that we can write

$$
k\left(\theta_{i}\right)=2\langle W\rangle \frac{\sin ^{2}\left(\theta_{i}\right)}{\delta z^{2}},
$$

where the angular dependence derives from the projection onto the axis of the secondary filament. Equations (5) and (6) represent a closed set of equations to calculate the configurationally averaged deformation energy $\langle W\rangle$ as a function of the number of cross-links $n$. In implementing this scheme, we have generated ensembles of filaments with a distribution of cross-linking angles as given by Eq. (1) and segment lengths according to Eq. (2). Note that there is no free parameter in this calculation. The equations are solved graphically in Fig. 2 by plotting both sides of Eq. (5) as a function of $\langle W\rangle$. The point of intersection, which solves the equation, is shown in the inset as a function of the number of cross-links $n$. For the same parameter window as used in the network simulations [15], it yields the scaling behavior of $\langle W\rangle \propto n^{5.75}$. This implies for the modulus the exponent of $\mu=6.75$, which improves upon the simple scaling picture presented above and provides a very accurate calculation of the scaling exponent $\mu$.

In conclusion, we have succeeded in deriving the macroscopic elasticity of random fibrous networks starting from a microscopic description of the displacement field in a manner that does not rely on the notion of affine deformations. We have given a floppy mode construction that may be applied to any two- or three-dimensional network with fibrous architecture, for example, paper or biological networks of semiflexible filaments. It may also be shown to be relevant to systems where the constraint of straight fibers is relaxed [26]. The unusually strong density dependence of the modulus found here is a consequence of the exponential segment length distribution Eq. (2) and the presence of the length scale $l_{\min }$. While identification of the floppy modes has been recognized to be highly important for a description of force transmission in granular media or the jamming transition in colloidal systems, one can rarely give the exact form of these zero-energy excitations. On the contrary, we have achieved an explicit construction of the floppy modes that can be put in the form of localized elementary excitations affecting only single filaments and their immediate surroundings.

It is a pleasure to acknowledge fruitful discussions with David Nelson and Mikko Alava.

[1] Soft and Fragile Matter, edited by M.E. Cates and M. R. Evans (Institute of Physics, London, 2000).

[2] A. Soloviev and A. Ismail-Zadeh, in Nonlinear Dynamics of the Lithosphere and Earthquake Prediction, edited by V.I. Keilis-Borok and A. Soloviev (Springer, Berlin, 2003).

[3] M. E. Cates, J. P. Wittmer, J.-P. Bouchaud, and P. Claudin, Phys. Rev. Lett. 81, 1841 (1998).

[4] E. I. Corwin, H. M. Jaeger, and S. Nagel, Nature (London) 435, 1075 (2005).

[5] S. Alexander, Phys. Rep. 296, 65 (1998).

[6] J. P. Wittmer, P. Claudin, M. E. Cates, and J.-P. Bouchaud, Nature (London) 382, 336 (1996).

[7] J.-P. Bouchaud, P. Claudin, D. Levine, and M. Otto, Eur. Phys. J. E 4, 451 (2001).

[8] A. Tanguy, J. P. Wittmer, F. Leonforte, and J.-L. Barrat, Phys. Rev. B 66, 174205 (2002).

[9] L. E. Silbert, A. J. Liu, and S. R. Nagel, Phys. Rev. Lett. 95, 098301 (2005).

[10] M. Wyart, S. R. Nagel, and T. A. Witten, Europhys. Lett. 72, 486 (2005); Ann. Phys. (Paris) 30, 1 (2005).

[11] D. J. Jacobs and M. F. Thorpe, Phys. Rev. Lett. 75, 4051 (1995).

[12] M. Alava and K. Niskanen, Rep. Prog. Phys. 69, 669 (2006).

[13] A. Bausch and K. Kroy, Nature Phys. 2, 231 (2006).

[14] C. Heussinger and E. Frey, Phys. Rev. Lett. 96, 017802 (2006); cond-mat/0512557.

[15] J. Wilhelm and E. Frey, Phys. Rev. Lett. 91, 108103 (2003).

[16] M. Latva-Kokko and J. Timonen, Phys. Rev. E 64, 066117 (2001).

[17] D. A. Head, A. J. Levine, and F. C. MacKintosh, Phys. Rev. Lett. 91, 108102 (2003).

[18] D. A. Head, A. J. Levine, and F. C. MacKintosh, Phys. Rev. E 68, 061907 (2003).

[19] P. R. Onck, T. Koeman, T. van Dillen, and E. van der Giessen, Phys. Rev. Lett. 95, 178102 (2005).

[20] B. A. DiDonna and T.C. Lubensky, Phys. Rev. E 72, 066619 (2005).

[21] O. Kallmes and H. Corte, Tappi 43, 737 (1960).

[22] Because of the finite fiber length, there are also two- and threefold coordinated cross-links.

[23] M. Kellomäki, J. Åström, and J. Timonen, Phys. Rev. Lett. 77, 2730 (1996).

[24] J. C. Maxwell, Philos. Mag. 27, 294 (1864).

[25] A similar construction holds in 3D, where, in addition, each cross-link acquires a floppy "out-of-plane" degree of freedom.

[26] C. Heussinger and E. Frey (to be published). 